\title{
Investigating the hidden-charm and hidden-bottom pentaquark resonances in scattering process
}

\author{
Hongxia Huang ${ }^{*}$ and Jialun Ping ${ }^{\dagger}$ \\ Department of Physics, Nanjing Normal University, Nanjing, Jiangsu 210097, China
}

(Received 25 November 2018; published 9 January 2019)

\begin{abstract}
In the framework of the quark delocalization color screening model, both the hidden-charm and hiddenbottom pentaquark resonances are studied in the hadron-hadron scattering process. A few narrow pentaquark resonances with a hidden-charm above $4.2 \mathrm{GeV}$ and some narrow pentaquark resonances with a hidden-bottom above $11 \mathrm{GeV}$ are found from corresponding scattering processes. In addition, the states $N \eta_{c}, N J / \psi, N \eta_{b}$, and $N \Upsilon$ with $I J^{P}=\frac{1}{2} \frac{1}{2}^{-}$and $N J / \psi$ and $N \Upsilon$ with $I J^{P}=\frac{1}{2} \frac{3}{2}^{-}$can all be bound by channelcoupling calculations. All of these heavy pentaquarks are worth investigating in future experiments.
\end{abstract}

DOI: $10.1103 /$ PhysRevD.99.014010

\section{INTRODUCTION}

In 2003 the LEPS Collaboration announced the observation of the pentaquark $\Theta^{+}[1]$, which inspired a lot of theoretical work and experimental searches for pentaquarks. However, this state was not confirmed by the subsequent more advanced experiments. Nevertheless, the LEPS Collaboration still insists on the existence of the pentaquark $\Theta^{+}$[2], and relevant experiment are in progress [3]. Moreover, there were also some theoretical studies on the existence of the hidden-charm pentaquarks [4-12]. In 2015, the claim of two hidden-charm pentaquark states $P_{c}(4380)$ and $P_{c}(4450)$ by the LHCb Collaboration [13] renewed the interest in pentaquarks and triggered further theoretical work on these two states. Current theoretical interpretations of $P_{c}(4380)$ and $P_{c}(4450)$ include baryon-meson molecules [14-24], diquark-triquark pentaquarks $[25,26]$, diquark-diquark-antiquark pentaquarks [27-30], genuine multiquark states [31], topological solitons [32], and kinematical threshold effects in the triangle singularity mechanism [33-35]. Lattice QCD simulations of $N J / \psi$ and $N \eta_{c}$ scattering were also performed to find these $P_{c}$ states [36]. Comprehensive discussions on current experimental progress and various theoretical interpretations of these candidates can be found in Ref. [37].

Recently, several works extended the study to the hidden-bottom sector to search for the hidden-bottom

\footnotetext{
*hxhuang@njnu.edu.cn

jlping@njnu.edu.cn
}

Published by the American Physical Society under the terms of the Creative Commons Attribution 4.0 International license. Further distribution of this work must maintain attribution to the author(s) and the published article's title, journal citation, and DOI. Funded by SCOAP ${ }^{3}$. pentaquark resonances. Reference [38] looked for the hidden-bottom pentaquark states with spin $3 / 2$ and $5 / 2$, and predictions for the masses might shed light on experimental searches for the bottom pentaquarks. Reference [39] studied both $\bar{D} \Lambda_{c}-\bar{D} \Sigma_{c}$ and $B \Lambda_{b}-B \Sigma_{b}$ interactions by using a unitary coupled-channel model, and several resonant and bound states with different spins and parities were dynamically generated in the mass range of the recently observed pentaquarks. The work of Ref. [39] indicated that there are more resonances for the $B \Lambda_{b}-B \Sigma_{b}$ interactions, and they are more tightly bound because the $b$-quark mass is larger than the $c$-quark mass. Reference [40] showed that standard constituent quark models produce hidden-charm pentaquarks $\bar{c} c q q q$, where $c$ denotes the charmed quark and $q$ is a light quark, and they also pointed out that the pentaquarks composed of $\bar{b} b q q q$ or $\bar{b} c q q q$ could be bound as well. Therefore, the hidden-bottom pentaquark resonances deserve further study.

To provide the necessary information for experiments to search for multiquark states, mass spectrum calculations alone are not enough. The calculation of hadron-hadron scattering, the main production process of multiquark states, is indispensable. The scattering phase shifts will show a resonance behavior in the resonance energy region. In many of the theoretical works mentioned above, $P_{c}(4380)$ and $P_{c}(4450)$ were investigated as bound states. In fact, these states will decay through the related open channels. As we mentioned in our previous work [18], in the bound-state calculation $P_{c}(4380)$ can be explained as the molecular pentaquark $\Sigma_{c}^{*} D$ with quantum number $J^{P}=\frac{3}{2}{ }^{-}$, but it can decay to the open channels $N J / \psi$ and $\Lambda_{c} D^{*}$. Therefore, we should study the $N J / \psi$ and $\Lambda_{c} D^{*}$ scattering process to check whether the $P_{c}(4380)$ is a resonance state. We performed a similar analysis for the dibaryon system, in which we obtained the $d^{*}$ resonance 
during the $N N$ scattering process and found that the energy and decay width of the partial wave of $N N$ were consistent with the experiment data [41]. Extending this work to the pentaquark system, we investigated the $N \phi$ state in the scattering channels $N \eta^{\prime}, \Lambda K$, and $\Sigma K$ [42]. Both the resonance mass and decay width were obtained, which provided the necessary information for experimental searches at Jefferson Lab. Therefore, it is interesting to extend such a study to the molecular pentaquarks with heavy quarks.

Generally, hadronic structure and hadron interactions belong to the low-energy physics of quantum chromodynamics (QCD), which are much harder to calculate directly from QCD because of the nonperturbative nature of QCD. One has to rely on effective theories and/or QCD-inspired models to get some insight into the phenomena of the hadronic world. The constituent quark model is one of them, which approximately transforms the complicated interactions between current quarks into dynamic properties of quasiparticles (constituent quarks) and considers the residual interactions between quasiparticles. There are various kinds of constituent quark models, such as the one-boson-exchange model, chiral quark model, quark delocalization color screening model (QDCSM), and so on. These models have been successful in describing the hadron spectrum, baryon-baryon interactions, and the bound state of two baryons, i.e., the deuteron. Among these phenomenological models the QDCSM, which was developed in the 1990s with the aim of explaining the similarities between nuclear (hadronic clusters of quarks) and molecular forces [43], was extensively used and studied by our group. In this model, quarks confined in one cluster are allowed to delocalize to a nearby cluster, and the confinement interaction between quarks in different clusters is modified to include a color screening factor.
The latter is a model description of the hidden color channel coupling effect [44]. The delocalization parameter is determined by the dynamics of the interacting multiquark system, and thus allows the system to choose the most favorable configuration through its own dynamics in a larger Hilbert space. This model was recently used to study the hidden-charm pentaquarks [18]. We found that the interaction between $\Sigma_{c}$ (or $\Sigma_{c}^{*}$ ) and $D$ (or $D^{*}$ ) was strong enough to form some bound states, and $P_{c}(4380)$ can be interpreted as the molecular state $\Sigma_{c}^{*} D$ with quantum numbers $I J^{P}=\frac{1}{2} \frac{3}{2}^{-}$. In this work, we continue to study the hidden-charm resonance states in the related hadronhadron scattering process. In addition, we extend the study to the hidden-bottom sector to search for hidden-bottom pentaquark resonances.

In the next section, the framework of the QDCSM and the calculation method are briefly introduced. Section III is devoted to the numerical results and discussions. A summary is given in the last section.

\section{QUARK MODEL AND THE CALCULATION METHOD}

Since our previous work on the bound-state calculation of the hidden-charm molecular pentaquark [18] was done using our QDCSM, we use the same model and parameters to study the pentaquark resonances here. Also, we use the well-developed resonating group method (RGM) to calculate the baryon-meson scattering phase shifts and to look for the resonance states [45].

\section{A. Quark delocalization color screening model}

The details of the QDCSM used in the present work can be found in Refs. [43,44,46]. Here, we just present the salient features of the model. The model Hamiltonian is

$$
\begin{aligned}
& H=\sum_{i=1}^{5}\left(m_{i}+\frac{p_{i}^{2}}{2 m_{i}}\right)-T_{c}+\sum_{i<j}\left[V^{G}\left(r_{i j}\right)+V^{\chi}\left(r_{i j}\right)+V^{C}\left(r_{i j}\right)\right] \\
& V^{G}\left(r_{i j}\right)=\frac{1}{4} \alpha_{s} \lambda_{i} \cdot \lambda_{j}\left[\frac{1}{r_{i j}}-\frac{\pi}{2}\left(\frac{1}{m_{i}^{2}}+\frac{1}{m_{j}^{2}}+\frac{4 \sigma_{i} \cdot \sigma_{j}}{3 m_{i} m_{j}}\right) \delta\left(r_{i j}\right)-\frac{3}{4 m_{i} m_{j} r_{i j}^{3}} S_{i j}\right] \text {, } \\
& V^{\chi}\left(r_{i j}\right)=\frac{1}{3} \alpha_{c h} \frac{\Lambda^{2}}{\Lambda^{2}-m_{\chi}^{2}} m_{\chi}\left\{\left[Y\left(m_{\chi} r_{i j}\right)-\frac{\Lambda^{3}}{m_{\chi}^{3}} Y\left(\Lambda r_{i j}\right)\right] \sigma_{i} \cdot \sigma_{j}+\left[H\left(m_{\chi} r_{i j}\right)-\frac{\Lambda^{3}}{m_{\chi}^{3}} H\left(\Lambda r_{i j}\right)\right] S_{i j}\right\} \mathbf{F}_{i} \cdot \mathbf{F}_{j}, \quad \chi=\pi, K, \eta, \\
& V^{C}\left(r_{i j}\right)=-a_{c} \lambda_{i} \cdot \lambda_{j}\left[f\left(r_{i j}\right)+V_{0}\right] \text {, } \\
& f\left(r_{i j}\right)= \begin{cases}r_{i j}^{2} & \text { if } i, \text { joccur in the same baryon orbit, } \\
\frac{1-e^{-\mu_{i j} r_{i j}^{2}}}{\mu_{i j}} & \text { if } i, \text { joccur in different baryon orbits, }\end{cases} \\
& S_{i j}=\frac{\left(\sigma_{i} \cdot \mathbf{r}_{i j}\right)\left(\sigma_{j} \cdot \mathbf{r}_{i j}\right)}{r_{i j}^{2}}-\frac{1}{3} \sigma_{i} \cdot \sigma_{j},
\end{aligned}
$$


where $S_{i j}$ is the quark tensor operator, $Y(x)$ and $H(x)$ are standard Yukawa functions [47], $T_{c}$ is the kinetic energy of the center of mass, $\alpha_{c h}$ is the chiral coupling constant determined as usual from the $\pi$-nucleon coupling constant, and $\alpha_{s}$ is the quark-gluon coupling constant. In order to cover the wide energy range from light to heavy quarks one introduces an effective scale-dependent quark-gluon coupling $\alpha_{s}(\mu)$ [48],

$$
\alpha_{s}(\mu)=\frac{\alpha_{0}}{\ln \left(\frac{\mu^{2}+u_{0}^{2}}{\Lambda_{0}^{2}}\right)},
$$

where $\mu$ is the reduced mass of the interacting quark pair. All other symbols have their usual meanings, and all parameters are taken from our previous work [18]. The quark delocalization in the QDCSM is realized by specifying the single-particle orbital wave function of the QDCSM as a linear combination of left and right Gaussians, which are the single-particle orbital wave functions used in the ordinary quark cluster model. One can refer to Ref. [18] to see the orbital wave functions.

\section{B. The calculation method}

Here, we calculate the baryon-meson scattering phase shifts and investigate the resonance states by using the RGM [45], a well-established method for studying boundstate and scattering problems. The wave function of the baryon-meson system is of the form

$$
\Psi=\mathcal{A}\left[\hat{\phi}_{A}\left(\boldsymbol{\xi}_{1}, \boldsymbol{\xi}_{2}\right) \hat{\phi}_{B}\left(\boldsymbol{\xi}_{3}\right) \chi_{L}\left(\boldsymbol{R}_{A B}\right)\right],
$$

where $\xi_{1}$ and $\xi_{2}$ are the internal coordinates for the baryon cluster $\mathrm{A}$, and $\boldsymbol{\xi}_{3}$ is the internal coordinate for the meson cluster $B . \boldsymbol{R}_{A B}=\boldsymbol{R}_{A}-\boldsymbol{R}_{B}$ is the relative coordinate between the two clusters. $\hat{\phi}_{A}$ and $\hat{\phi}_{B}$ are the internal cluster wave functions of the baryon $A$ (antisymmetrized) and meson $B$, and $\chi_{L}\left(\boldsymbol{R}_{A B}\right)$ is the relative-motion wave function between two clusters. The symbol $\mathcal{A}$ is the antisymmetrization operator defined as

$$
\mathcal{A}=1-P_{14}-P_{24}-P_{34},
$$

where 1,2, and 3 stand for the quarks in the baryon cluster and 4 stands for the quark in the meson cluster. For a boundstate problem, $\chi_{L}\left(\boldsymbol{R}_{A B}\right)$ is expanded in Gaussian bases,

$$
\begin{aligned}
\chi_{L}\left(\boldsymbol{R}_{A B}\right)= & \frac{1}{\sqrt{4 \pi}}\left(\frac{6}{5 \pi b^{2}}\right)^{3 / 4} \sum_{i=1}^{n} C_{i} \\
& \times \int \exp \left[-\frac{3}{5 b^{2}}\left(\boldsymbol{R}_{A B}-\boldsymbol{S}_{i}\right)^{2}\right] Y_{L M}\left(\hat{\boldsymbol{S}_{i}}\right) d \hat{\boldsymbol{S}_{i}} \\
= & \sum_{i=1}^{n} C_{i} \frac{u_{L}\left(R_{A B}, S_{i}\right)}{R_{A B}} Y_{L M}\left(\hat{\boldsymbol{R}}_{A B}\right),
\end{aligned}
$$

with

$$
\begin{aligned}
u_{L}\left(R_{A B}, S_{i}\right)= & \sqrt{4 \pi}\left(\frac{6}{5 \pi b^{2}}\right)^{3 / 4} R_{A B} \exp \left[-\frac{3}{5 b^{2}}\left(R_{A B}^{2}-S_{i}^{2}\right)\right] \\
& \times i^{L} j_{L}\left(-i \frac{6}{5 b^{2}} R_{A B} S_{i}\right),
\end{aligned}
$$

where $S_{i}$ is the generating coordinate, $C_{i}$ are the expansion coefficients, $n$ is the number of Gaussian bases (which is determined by the stability of the results), and $j_{L}$ is the $L$ th spherical Bessel function.

For a scattering problem, the relative wave function is expanded as

$$
\chi_{L}\left(\boldsymbol{R}_{A B}\right)=\sum_{i=1}^{n} C_{i} \frac{\tilde{u}_{L}\left(R_{A B}, S_{i}\right)}{R_{A B}} Y_{L M}\left(\hat{\boldsymbol{R}}_{A B}\right),
$$

with

$$
\begin{aligned}
& \tilde{u}_{L}\left(R_{A B}, S_{i}\right) \\
& \quad= \begin{cases}\alpha_{i} u_{L}\left(R_{A B}, S_{i}\right), & R_{A B} \leq R_{C}, \\
{\left[h_{L}^{-}\left(k_{A B}, R_{A B}\right)-s_{i} h_{L}^{+}\left(k_{A B}, R_{A B}\right)\right] R_{A B},} & R_{A B} \geq R_{C},\end{cases}
\end{aligned}
$$

where $h_{L}^{ \pm}$is the $L$ th spherical Hankel function, $k_{A B}$ is the momentum of relative motion with $k_{A B}=\sqrt{2 \mu_{A B} E_{\mathrm{cm}}}, \mu_{A B}$ is the reduced mass of the two hadrons $(A$ and $B)$ of the open channel, $E_{\mathrm{cm}}$ is the incident energy, and $R_{C}$ is a cutoff radius beyond which all of the strong interactions can be disregarded. $\alpha_{i}$ and $s_{i}$ are complex parameters which are determined by the smoothness condition at $R_{A B}=R_{C}$, and $C_{i}$ satisfy $\sum_{i=1}^{n} C_{i}=1$. After performing the variational procedure, the $L$ th partial-wave equation for the scattering problem can be deduced as

$$
\sum_{j=1}^{n} \mathcal{L}_{i j}^{L} C_{j}=\mathcal{M}_{i}^{L} \quad(i=0,1, \ldots, n-1)
$$

with

$$
\begin{gathered}
\mathcal{L}_{i j}^{L}=\mathcal{K}_{i j}^{L}-\mathcal{K}_{i 0}^{L}-\mathcal{K}_{0 j}^{L}+\mathcal{K}_{00}^{L}, \\
\mathcal{M}_{i}^{L}=\mathcal{K}_{00}^{L}-\mathcal{K}_{i 0}^{L},
\end{gathered}
$$

and

$$
\begin{aligned}
\mathcal{K}_{i j}^{L}= & \left\langle\hat{\phi}_{A}\left(\boldsymbol{\xi}_{1}^{\prime}, \boldsymbol{\xi}_{2}^{\prime}\right) \hat{\phi}_{B}\left(\boldsymbol{\xi}_{3}^{\prime}\right) \frac{\tilde{u}_{L}\left(R_{A B}^{\prime}, S_{i}\right)}{R_{A B}^{\prime}} Y_{L M}\left(\hat{\boldsymbol{R}}_{A B}^{\prime}\right)\right. \\
& |H-E| \\
& \left.\mathcal{A}\left[\hat{\phi}_{A}\left(\boldsymbol{\xi}_{1}, \boldsymbol{\xi}_{2}\right) \hat{\phi}_{B}\left(\boldsymbol{\xi}_{3}\right) \frac{\tilde{u}_{L}\left(R_{A B}, S_{j}\right)}{R_{A B}} Y_{L M}\left(\hat{\boldsymbol{R}}_{A B}\right)\right]\right\rangle .
\end{aligned}
$$


By solving Eq. (9) we obtain the expansion coefficients $C_{i}$. Then, the $S$ matrix element $S_{L}$ and the phase shifts $\delta_{L}$ are given by

$$
S_{L} \equiv e^{2 i \delta_{L}}=\sum_{i=1}^{n} C_{i} s_{i}
$$

Resonances are unstable particles that are usually observed as bell-shaped structures in the scattering cross sections of their open channels. The fundamental properties of a simple narrow resonance correspond to the visible cross section features: the mass $M$ is at the peak position, and the decay width $\Gamma$ is the half-width of the bell shape. The cross section $\sigma_{L}$ and the scattering phase shifts $\delta_{L}$ are related by

$$
\sigma_{L}=\frac{4 \pi}{k^{2}} \cdot(2 l+1) \cdot \sin ^{2} \delta_{L},
$$

where $k=\sqrt{2 \mu E_{\mathrm{cm}}} / \hbar ;, \mu$ is the reduced mass of the two hadrons of the open channel, and $E_{\mathrm{cm}}$ is the incident energy. Therefore, resonances can also usually be observed in the scattering phase shift, where the phase shift of the scattering channels rises through $\frac{\pi}{2}$ at a resonance mass. We can obtain a resonance mass at the position of the phase shift of $\frac{\pi}{2}$. The decay width is the mass difference between the phase shift of $\frac{3 \pi}{4}$ and $\frac{\pi}{4}$.

\section{RESULTS AND DISCUSSIONS}

From our previous bound-state calculation [18] for the $I J^{P}=\frac{1}{2} \frac{1}{2}$ system, the single channels $\Sigma_{c} D, \Sigma_{c} D^{*}$, and $\Sigma_{c}^{*} D^{*}$ were bound, while the other four channels $N \eta_{c}$, $N J / \psi, \Lambda_{c} D$, and $\Lambda_{c} D^{*}$ were unbound and scattering channels. The case is similar for the $I J^{P}=\frac{1}{2} \frac{3}{2}-$ system: there are two scattering channels $\left(N J / \psi\right.$ and $\left.\Lambda_{c} D^{*}\right)$ and three bound-state channels $\left(\Sigma_{c} D^{*}, \Sigma_{c}^{*} D\right.$, and $\left.\Sigma_{c}^{*} D^{*}\right)$. These bound states may appear as resonance states in the corresponding scattering channels. We should mention that all of the states that we study here are in the $S$ wave because we found that there was no bound state with higher partial waves in our calculations. The $S$-wave bound states that decay to $D$-wave open channels through tensor interactions are neglected here due to their small decay widths, so the total decay widths of the states given below are the lower limits, as only the hidden-charm channels are considered in this work. We analyze two types of channel coupling in this work. The first is the two-channel coupling with a single bound state and a related open channel, while the other is the four-channel coupling with three bound states and a corresponding open channel. The general features of the calculated results are as follows.

For the $I J^{P}=\frac{1}{2} \frac{1}{2}$ system, we first calculate the twochannel coupling. The phase shifts of all scattering channels are shown in Fig. 1. The phase shifts of the $N \eta_{c}$ channel [see Fig. 1(a)] clearly show three resonance states, which means that every bound state $\Sigma_{c} D, \Sigma_{c} D^{*}$, and $\Sigma_{c}^{*} D^{*}$

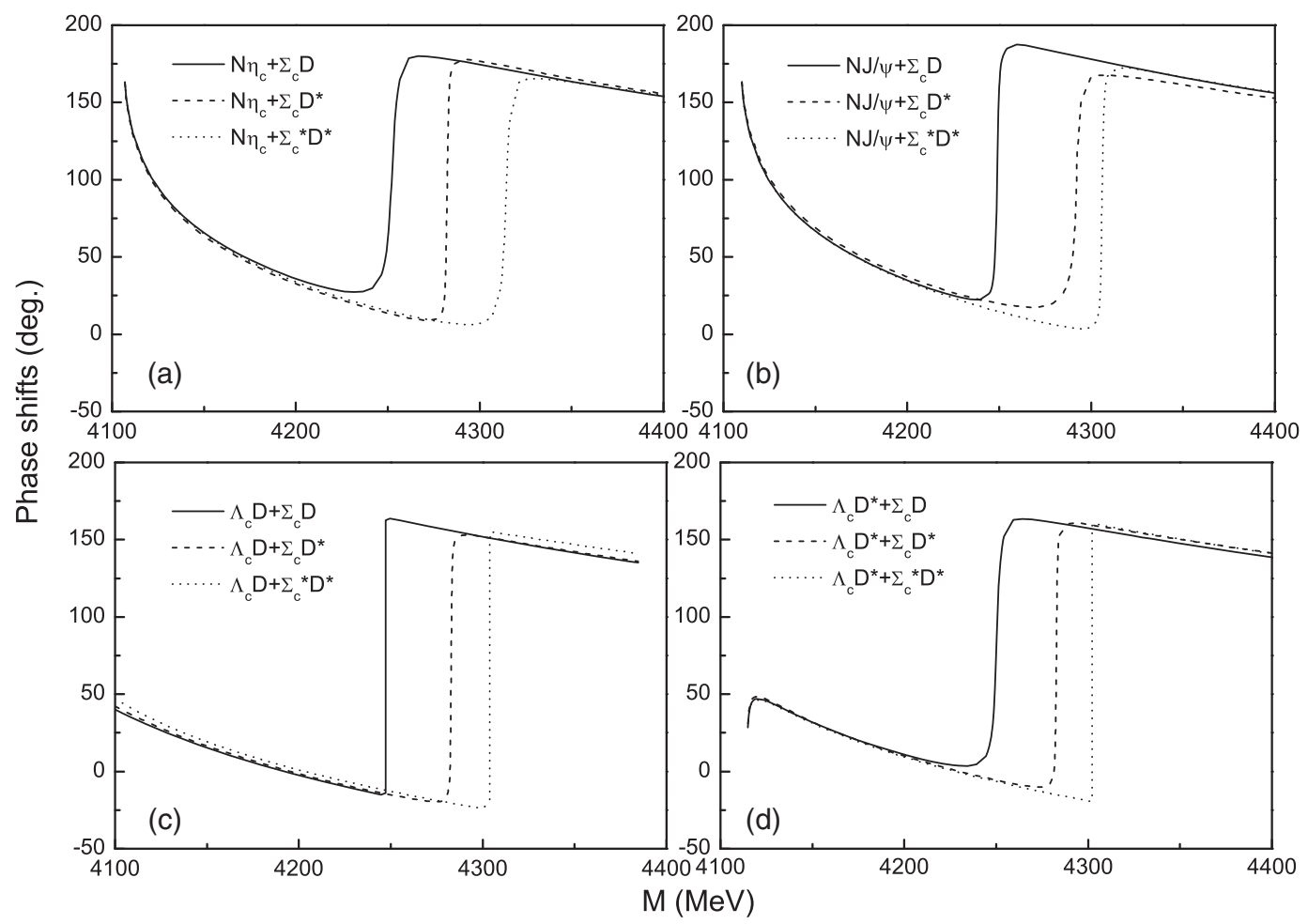

FIG. 1. The $N \eta_{c}, N J / \psi, \Lambda_{c} D$, and $\Lambda_{c} D^{*} S$-wave phase shifts with two-channel coupling for the $I J^{P}=\frac{1}{2} \frac{1}{2}{ }^{-}$system. 
TABLE I. The theoretical and experimental thresholds (in $\mathrm{MeV}$ ) of the states used in this work.

\begin{tabular}{lllllllll}
\hline \hline & $N \eta_{c}$ & $N J / \psi$ & $\Lambda_{c} D$ & $\Lambda_{c} D^{*}$ & $\Sigma_{c} D$ & $\Sigma_{c} D^{*}$ & $\Sigma_{c}^{*} D$ & $\Sigma_{c}^{*} D^{*}$ \\
\hline$M_{\text {theo }}$ & 4106.5 & 4109.5 & 4079.8 & 4114.3 & 4257.8 & 4292.4 & 4283.7 & 4318.3 \\
$M_{\text {exp }}$ & 3919 & 4035 & 4150 & 4293 & 4319 & 4462 & 4384 & 4572 \\
\hline & $N \eta_{b}$ & $N \Upsilon$ & $\Lambda_{b} B$ & $\Lambda_{b} B^{*}$ & $\Sigma_{b} B$ & $\Sigma_{b} B^{*}$ & $\Sigma_{b}^{*} B$ & $\Sigma_{b}^{*} B^{*}$ \\
\hline$M_{\text {theo }}$ & 10977.4 & 10977.6 & 10940.7 & 10951.5 & 11130.6 & 11141.4 & 11138.7 & 11149.6 \\
$M_{\exp }$ & 10330 & 10399 & 10898 & 10944 & 11090 & 11136 & 11111 & 11157 \\
\hline \hline
\end{tabular}

TABLE II. The masses and decay widths (in $\mathrm{MeV}$ ) of the $I J^{P}=\frac{1}{2} \frac{1}{2}^{-}$resonance states in the $N \eta_{c}, N J / \psi, \Lambda_{c} D$, and $\Lambda_{c} D^{*} S$-wave scattering process.

\begin{tabular}{|c|c|c|c|c|c|c|c|c|c|c|c|c|}
\hline & \multicolumn{6}{|c|}{ Two-channel coupling } & \multicolumn{6}{|c|}{ Four-channel coupling } \\
\hline & \multicolumn{2}{|c|}{$\Sigma_{c} D$} & \multicolumn{2}{|c|}{$\Sigma_{c} D^{*}$} & \multicolumn{2}{|c|}{$\Sigma_{c}^{*} D^{*}$} & \multicolumn{2}{|c|}{$\Sigma_{c} D$} & \multicolumn{2}{|c|}{$\Sigma_{c} D^{*}$} & \multicolumn{2}{|c|}{$\Sigma_{c}^{*} D^{*}$} \\
\hline & $M^{\prime}$ & $\Gamma_{i}$ & $M^{\prime}$ & $\Gamma_{i}$ & $M^{\prime}$ & $\Gamma_{i}$ & $M^{\prime}$ & $\Gamma_{i}$ & $M^{\prime}$ & $\Gamma_{i}$ & $M^{\prime}$ & $\Gamma_{i}$ \\
\hline$N \eta_{c}$ & 4312.9 & 6.0 & 4451.7 & 1.1 & 4523.1 & 3.5 & 4311.3 & 4.5 & 4448.8 & 1.0 & 4525.8 & 4.0 \\
\hline$N J / \psi$ & 4309.9 & 2.0 & 4461.6 & 4.0 & 4514.7 & 1.2 & 4307.9 & 1.2 & 4459.7 & 3.9 & $\mathrm{nr}$ & $\ldots$ \\
\hline$\Lambda_{c} D$ & 4308.4 & 0.003 & 4452.6 & 1.0 & 4512.6 & 0.004 & 4306.7 & 0.02 & 4461.6 & 1.0 & $\mathrm{nr}$ & $\ldots$ \\
\hline$\Lambda_{c} D^{*}$ & 4311.6 & 3.5 & 4452.5 & 1.0 & 4510.8 & 0.005 & 4307.7 & 1.4 & 4449.0 & 0.3 & $\mathrm{nr}$ & $\cdots$ \\
\hline$\Gamma_{\text {total }}$ & & 11.5 & & 7.1 & & 4.7 & & 7.1 & & 6.2 & & 4.0 \\
\hline
\end{tabular}

appears as a resonance state by coupling to the scattering channel $N \eta_{c}$. The other scattering channels $N J / \psi, \Lambda_{c} D$, and $\Lambda_{c} D^{*}$ [see Figs. 1(b)-1(d)] show similar results as for $N \eta_{c}$. The resonance mass and decay width of every resonance state can be obtained from the shape of the resonance. Here, we should note that the horizontal axis $M$ in Fig. 1 is the sum of the corresponding theoretical threshold of the open channel and the incident energy, so the mass at the position of the phase shift of $\frac{\pi}{2}$ is the theoretical resonance mass of the corresponding resonance state. In order to minimize the theoretical errors and to compare calculated results to the experimental data, we shift the mass of the

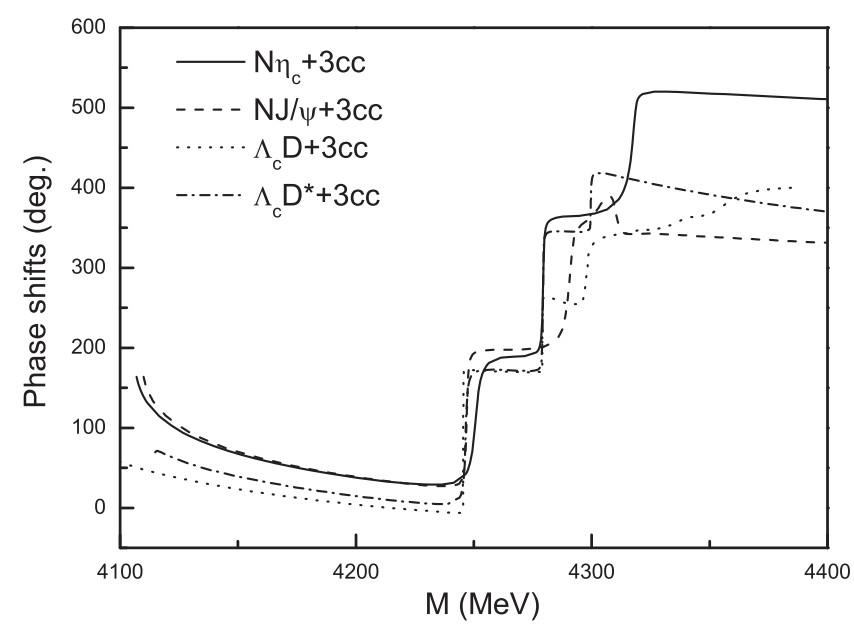

FIG. 2. The $N \eta_{c}, N J / \psi, \Lambda_{c} D$, and $\Lambda_{c} D^{*} S$-wave phase shifts with four-channel coupling for the $I J^{P}=\frac{1}{2} \frac{1}{2}$ system. resonance state to $M^{\prime}=M-M_{\text {theo }}+M_{\text {exp }}$, where $M_{\text {theo }}$ and $M_{\text {exp }}$ are the theoretical and experimental thresholds of the resonance state, respectively. Taking the resonance state $\Sigma_{c} D$ in the $N \eta_{c}$ channel as an example, the resonance mass

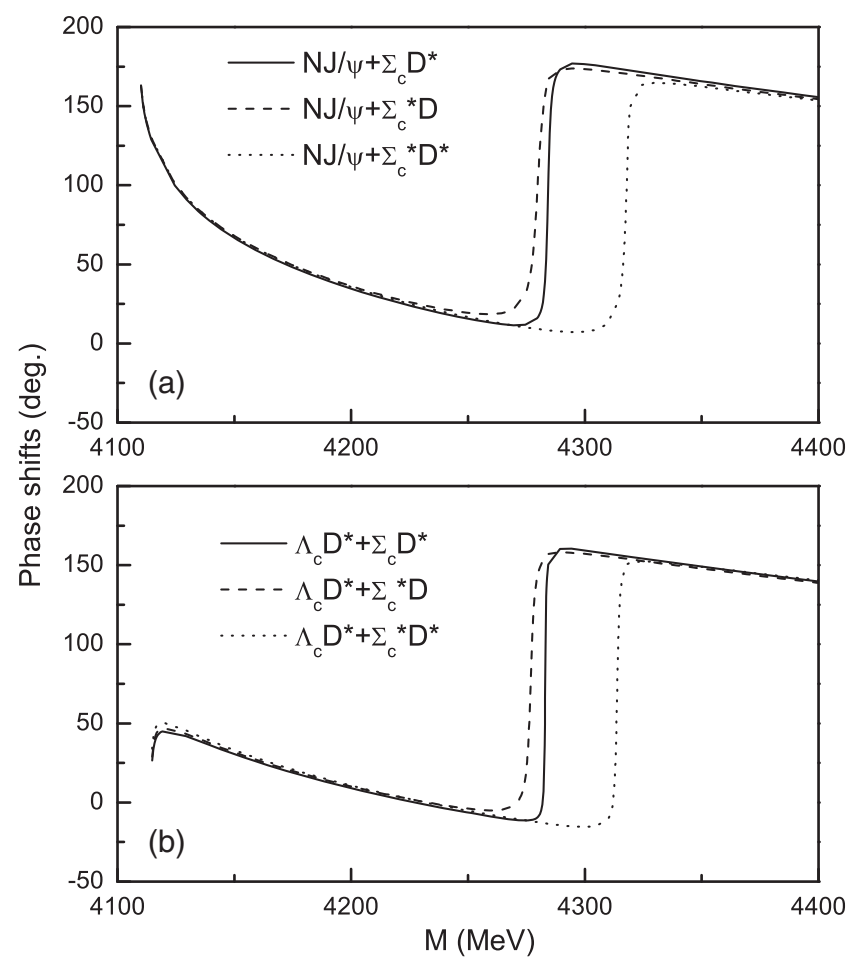

FIG. 3. The $N J / \psi$ and $\Lambda_{c} D^{*} S$-wave phase shifts with twochannel coupling for the $I J^{P}=\frac{1}{2} \frac{3}{2}-$ system. 


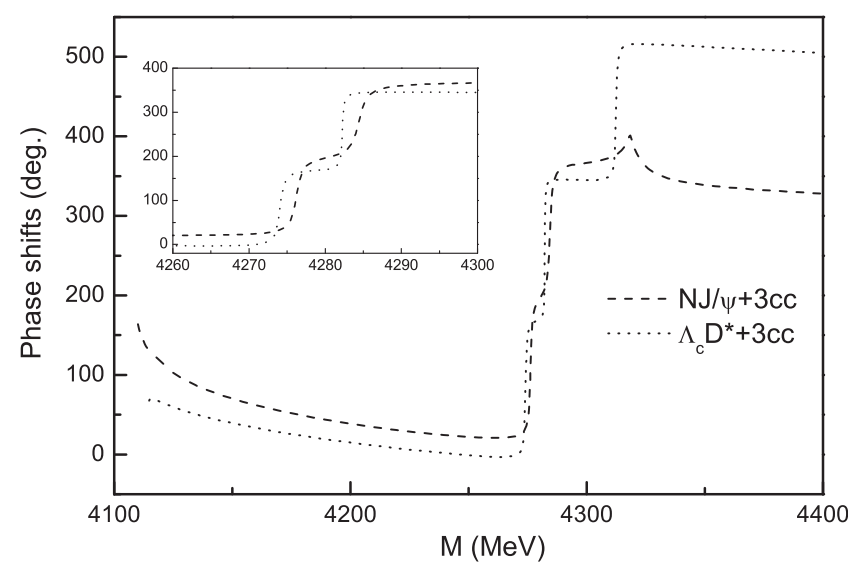

FIG. 4. The $N J / \psi$ and $\Lambda_{c} D^{*} S$-wave phase shifts with fourchannel coupling for the $I J^{P}=\frac{1}{2} \frac{3}{2}^{-}$system.

shown in Fig. 1(a) is $M=4251.7 \mathrm{MeV}$, the theoretical threshold is $M_{\text {theo }}=4257.8 \mathrm{MeV}$, and the experimental threshold is $M_{\exp }=4319 \mathrm{MeV}$. Then, the final resonance mass $M^{\prime}=4251.7-4257.8+4319=4312.9 \mathrm{MeV}$.

All of the theoretical and experimental thresholds of the states used in this work are shown in Table I. The resonance masses and decay widths are listed in Table II, where $M^{\prime}$ is the resonance mass, $\Gamma_{i}$ is the partial decay width of the resonance state decaying to a different open channel, and $\Gamma_{\text {total }}$ is the total decay width of the resonance state in the
TABLE III. The masses and decay widths (in $\mathrm{MeV}$ ) of the $I J^{P}=\frac{1}{2} \frac{3}{2}-$ resonance states in the $N J / \psi$ and $\Lambda_{c} D^{*} S$-wave scattering process.

\begin{tabular}{|c|c|c|c|c|c|c|}
\hline & \multicolumn{6}{|c|}{ Two-channel coupling } \\
\hline & \multicolumn{2}{|c|}{$\Sigma_{c} D^{*}$} & \multicolumn{2}{|c|}{$\Sigma_{c}^{*} D$} & \multicolumn{2}{|c|}{$\sum_{c}^{*} D^{*}$} \\
\hline & $M^{\prime}$ & $\Gamma_{i}$ & $M^{\prime}$ & $\Gamma_{i}$ & $M^{\prime}$ & $\Gamma_{i}$ \\
\hline$\overline{N J / \psi}$ & 4453.8 & 1.7 & 4379.7 & 4.5 & 4526.4 & 2.5 \\
\hline$\Lambda_{c} D^{*}$ & 4452.7 & 0.8 & 4377.6 & 3.2 & 4522.7 & 1.8 \\
\hline \multirow[t]{4}{*}{$\underline{\Gamma_{\text {total }}}$} & & 2.5 & & 7.7 & & 4.3 \\
\hline & \multicolumn{6}{|c|}{ Four-channel coupling } \\
\hline & \multicolumn{2}{|c|}{$\Sigma_{c} D^{*}$} & \multicolumn{2}{|c|}{$\Sigma_{c}^{*} D$} & \multicolumn{2}{|c|}{$\sum_{c}^{*} D^{*}$} \\
\hline & $M^{\prime}$ & $\Gamma_{i}$ & $M^{\prime}$ & $\Gamma_{i}$ & $M^{\prime}$ & $\Gamma_{i}$ \\
\hline$N J / \psi$ & 4445.7 & 1.5 & 4376.4 & 1.5 & $\mathrm{nr}$ & . \\
\hline$\Lambda_{c} D^{*}$ & 4444.0 & 0.3 & 4374.4 & 0.9 & 4523.0 & 1.0 \\
\hline$\Gamma_{\text {total }}$ & & 1.8 & & 2.4 & & 1.0 \\
\hline
\end{tabular}

present work. Compared to the results of our previous bound-state calculation [18], the mass shift of every resonance state is not very large, which indicates that the scattering channel and bound-state channel coupling effect is not very strong, although the coupling is through the central part of the quark-quark interactions. The reason

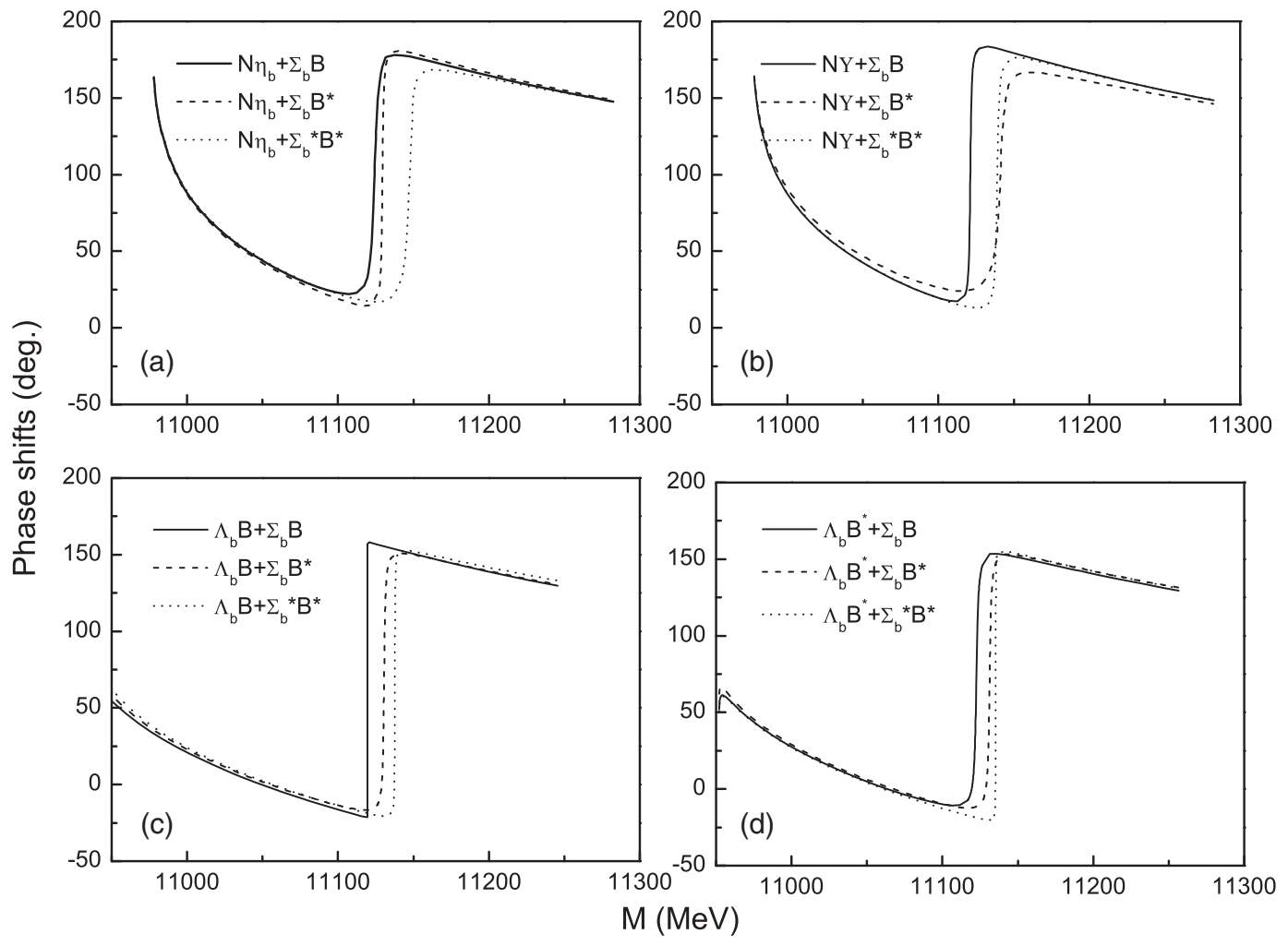

FIG. 5. The $N \eta_{b}, N \Upsilon, \Lambda_{b} B$, and $\Lambda_{b} B^{*} S$-wave phase shifts with two-channel coupling for the $I J^{P}=\frac{1}{2} \frac{1}{2}$ system. 


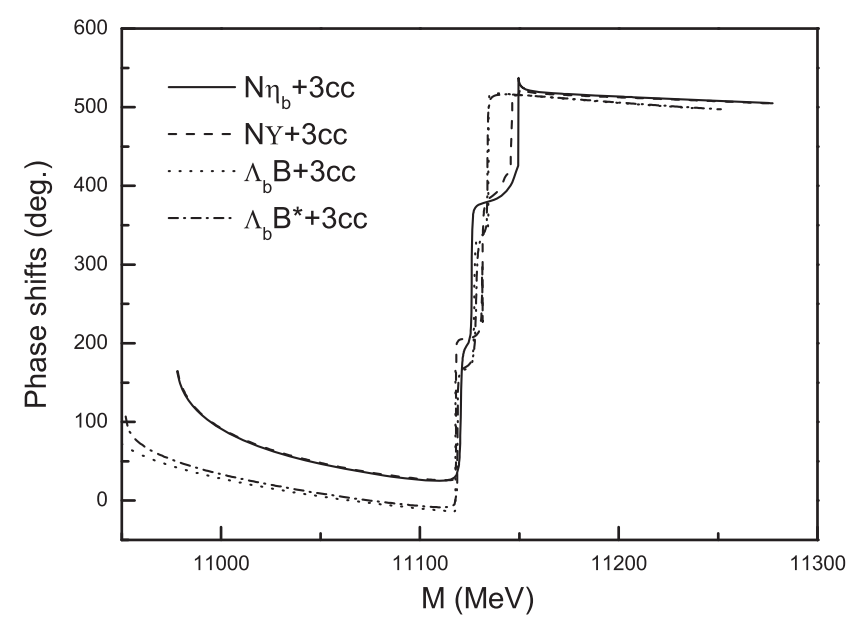

FIG. 6. The $N \eta_{b}, N \Upsilon, \Lambda_{b} B$ and $\Lambda_{b} B^{*} S$-wave phase shifts with four-channel coupling for the $I J^{P}=\frac{1}{2} \frac{1}{2}$ - system.

is that the mass difference between the scattering channel and the bound-state channel is large, about $100-400 \mathrm{MeV}$.

To investigate the effect of the channel coupling of the bound states, we also calculate the four-channel coupling. The phase shifts of all scattering channels of the $I J^{P}=\frac{1}{2} \frac{1}{2}-$ system are shown in Fig. 2, which shows a multiresonance behavior. There are three resonance states in the $N \eta_{c}$ scattering phase shifts corresponding to $\Sigma_{c} D, \Sigma_{c} D^{*}$, and $\Sigma_{c}^{*} D^{*}$, while in other scattering channels there are only two resonance states corresponding to $\Sigma_{c} D$ and $\Sigma_{c} D^{*}$. There is only a cusp around the threshold of the third state, $\Sigma_{c}^{*} D^{*}$. The reason is that the channel coupling pushes the higher state above the threshold. The resonance masses and decay widths of resonance states with four-channel coupling are also listed in Table II. Both $\Sigma_{c} D$ and $\Sigma_{c} D^{*}$ with $I J^{P}=\frac{1}{2} \frac{1}{2}-$ are resonance states in related scattering channels. The resonance mass range of the $\Sigma_{c} D$ state is 4306.74311.3 MeV and the total decay width is about 7.1 MeV, and $\Sigma_{c} D^{*}$ has a mass range of $4448.8-$ $4461.6 \mathrm{MeV}$ and a total decay width of $6.2 \mathrm{MeV}$. $\Sigma_{c}^{*} D^{*}$ appears as a resonance state only in the $N \eta_{c}$ channel, with a mass of $4525.8 \mathrm{MeV}$ and a decay width of $4.0 \mathrm{MeV}$. These results are qualitatively similar to the conclusions of Ref. [4], which predicted two new $N^{*}$ states [the $\Sigma_{c} D$ molecular state $N^{*}(4265)$ and the $\Sigma_{c} D^{*}$ molecular state $\left.N^{*}(4415)\right]$ in the coupled-channel unitary approach.

In Figs. 1 and 2 the low-energy scattering phase shifts of both the $N \eta_{c}$ and $N J / \psi$ channels go to $180^{\circ}$ at $E_{\text {c.m. }} \sim 0$ and rapidly decrease as $E_{\text {c.m. }}$ increases, which implies that both the $N \eta_{c}$ and $N J / \psi$ states are bound states with the help of channel coupling. Meanwhile, the slope of the lowenergy phase shifts (near $E_{\mathrm{c} . \mathrm{m} .} \sim 0$ ) of both $\Lambda_{c} D$ and $\Lambda_{c} D^{*}$ is opposite to that of the $N \eta_{c}$ and $N J / \psi$ channels, which means that neither the $\Lambda_{c} D$ nor $\Lambda_{c} D^{*}$ state is a bound state even with channel coupling. All of these results are consistent with our bound-state calculation [18].

We perform the same calculation for the $I J^{P}=\frac{1}{2} \frac{3}{2}-$ system and similar results are obtained. In the two-channel coupling calculation, three bound states $\Sigma_{c} D^{*}, \Sigma_{c}^{*} D$, and $\Sigma_{c}^{*} D^{*}$ appear as resonance states in the scattering phase shifts $N J / \psi$ and $\Lambda_{c} D^{*}$, which are shown in Fig. 3. In the four-channel coupling calculation, the multiresonance behavior appears again, as shown in Fig. 4. Three resonance states appear in the $\Lambda_{c} D^{*}$ scattering phase shifts corresponding to $\Sigma_{c} D^{*}, \Sigma_{c}^{*} D$, and $\Sigma_{c}^{*} D^{*}$, while in the $N J / \psi$ scattering channels there are only two resonance states corresponding to $\Sigma_{c} D^{*}$ and $\Sigma_{c}^{*} D$. The cusp in the dashed line of Fig. 4 is a remnant of the $\Sigma_{c}^{*} D^{*}$. The resonance masses and decay widths of resonance states using two kinds of channel coupling are listed in Table III. The $\Sigma_{c} D^{*}$ is shown as a resonance state in both the $N J / \psi$ and $\Lambda_{c} D^{*}$ scattering processes with a mass range of 4452.0$4454.0 \mathrm{MeV}$ and a decay width of $1.8 \mathrm{MeV}$; the $\Sigma_{c}^{*} D$ is also a resonance state in both the $N J / \psi$ and $\Lambda_{c} D^{*}$ scattering channels with a mass range of 4374.44376.4 MeV and a decay width of $2.4 \mathrm{MeV}$; and $\Sigma_{c}^{*} D^{*}$ appears as a resonance state only in the $\Lambda_{c} D^{*}$ channel with a mass of $4523.0 \mathrm{MeV}$ and a decay width of $1.0 \mathrm{MeV}$. It is obvious that the mass of the resonance state $\Sigma_{c}^{*} D$ is consistent with the $P c(4380)$, but the decay width is much smaller than the experimental data, which is about $200 \mathrm{MeV}$. As mentioned above, only the hidden-charm channels are considered in this work, so here the total decay

TABLE IV. The masses and decay widths (in MeV) of the $I J^{P}=\frac{1}{2} \frac{1}{2}$ - resonance states in the $N \eta_{b}, N \Upsilon, \Lambda_{b} B$, and $\Lambda_{b} B^{*} S$-wave scattering process.

\begin{tabular}{|c|c|c|c|c|c|c|c|c|c|c|c|c|}
\hline & \multicolumn{6}{|c|}{ Two-channel coupling } & \multicolumn{6}{|c|}{ Four-channel coupling } \\
\hline & \multicolumn{2}{|c|}{$\Sigma_{b} B$} & \multicolumn{2}{|c|}{$\Sigma_{b} B^{*}$} & \multicolumn{2}{|c|}{$\Sigma_{b}^{*} B^{*}$} & \multicolumn{2}{|c|}{$\Sigma_{b} B$} & \multicolumn{2}{|c|}{$\Sigma_{b} B^{*}$} & \multicolumn{2}{|c|}{$\Sigma_{b}^{*} B^{*}$} \\
\hline & $M^{\prime}$ & $\Gamma_{i}$ & $M^{\prime}$ & $\Gamma_{i}$ & $M^{\prime}$ & $\Gamma_{i}$ & $M^{\prime}$ & $\Gamma_{i}$ & $M^{\prime}$ & $\Gamma_{i}$ & $M^{\prime}$ & $\Gamma_{i}$ \\
\hline$N \eta_{b}$ & 11083.3 & 4.0 & 11123.9 & 1.4 & 11154.5 & 4.7 & 11079.8 & 1.2 & 11120.6 & 0.4 & 11156.9 & 2.0 \\
\hline$N \Upsilon$ & 11080.4 & 1.4 & 11135.4 & 6.6 & 11146.2 & 2.0 & 11077.5 & 0.1 & 11125.8 & 0.8 & 11153.5 & 3.0 \\
\hline$\Lambda_{b} B$ & 11079.0 & 0.0003 & 11125.4 & 2.0 & 11145.1 & 0.49 & 11077.2 & 0.001 & 11122.0 & 0.6 & 11141.8 & 0.1 \\
\hline$\Lambda_{b} B^{*}$ & 11082.2 & 2.6 & 11126.2 & 2.3 & 11142.7 & 0.22 & 11078.3 & 0.3 & 11123.0 & 1.2 & 11141.5 & 0.4 \\
\hline$\Gamma_{\text {total }}$ & & 7.0 & & 12.3 & & 7.4 & & 1.6 & & 3.0 & & 5.5 \\
\hline
\end{tabular}




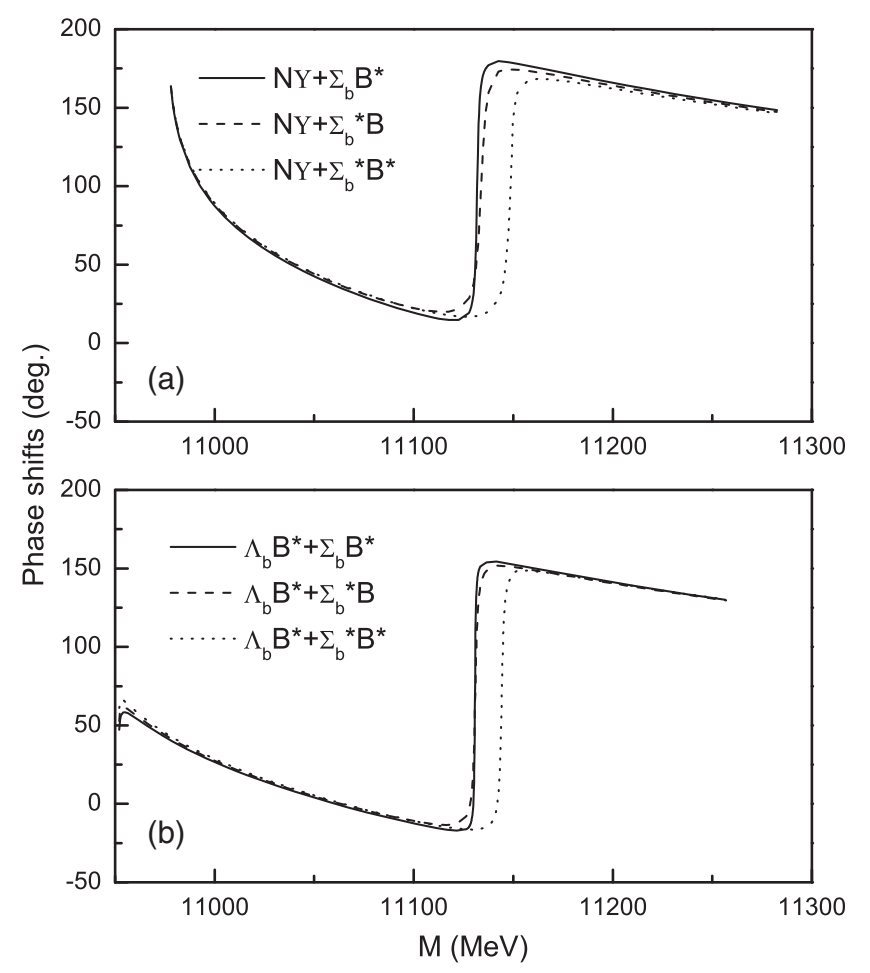

FIG. 7. The $N \Upsilon$ and $\Lambda_{b} B^{*} S$-wave phase shifts with twochannel coupling for the $I J^{P}=\frac{1}{2} \frac{3}{2}{ }^{-}$system.

widths of these states are the lower limits. More decay channels should be considered in future work.

Moreover, the behavior of the low-energy phase shifts of the $N J / \psi$ channel in both Figs. 3 and 4 is similar to that in Figs. 1 and 2. This indicates that the $N J / \psi$ channel with $I J^{P}=\frac{1}{2} \frac{3}{2}$ can be bound by channel-coupling calculations. By contrast, the slope of the low-energy phase shifts of $\Lambda_{c} D^{*}$ is opposite to that of the $N J / \psi$ channel, which means that $\Lambda_{c} D^{*}$ is unbound even with channel coupling. All of these results are also consistent with our previous boundstate calculation in Ref. [18].

Because of the heavy flavor symmetry, we also extend the study to the hidden-bottom pentaquarks. The results are similar to those for the hidden-charm molecular pentaquarks. From Figs. 5 and 6 , we can see that the $\Sigma_{b} B, \Sigma_{b} B^{*}$, and $\Sigma_{b}^{*} B^{*}$ states with $I J^{P}=\frac{1}{2} \frac{1}{2}{ }^{-}$appear as resonance states in all scattering channels $\left(N \eta_{b}, N \Upsilon, \Lambda_{b} B\right.$, and $\left.\Lambda_{b} B^{*}\right)$. The masses and decay widths are given in Table IV. The resonance mass range of the $\Sigma_{b} B$ state is $11077.2-11$ $079.8 \mathrm{MeV}$ and its decay width is about $1.6 \mathrm{MeV} ; \Sigma_{b} B^{*}$ has a mass range of $11120.6-11125.8 \mathrm{MeV}$ and a decay width of $3.0 \mathrm{MeV}$; and $\Sigma_{b}^{*} B^{*}$ has a mass range of $11141.5-$ $11156.9 \mathrm{MeV}$ and a decay width of 5.6 MeV. These results are qualitatively similar to the conclusions of Ref. [49], which predicted a few narrow $N^{*}$ resonances with hidden beauty around $11 \mathrm{GeV}$ in the coupled-channel unitary approach.

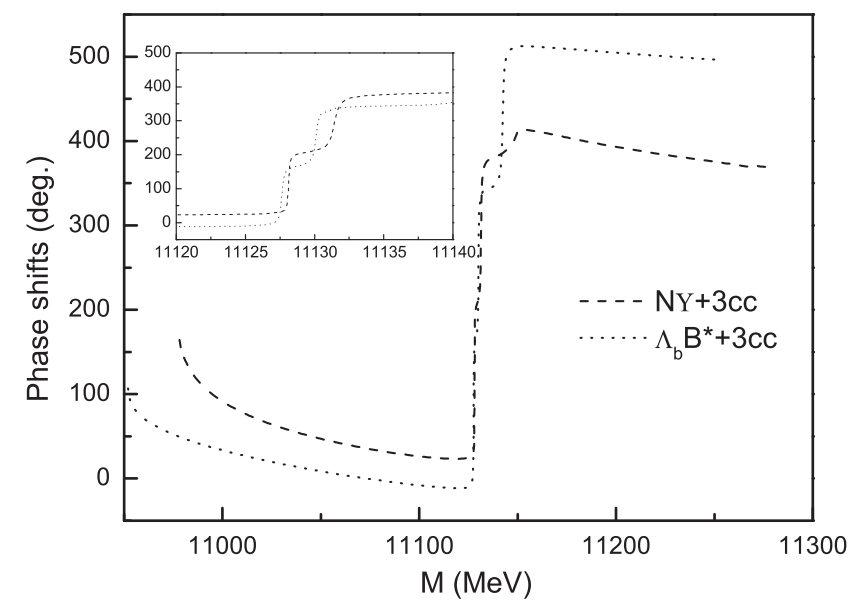

FIG. 8. The $N \Upsilon$ and $\Lambda_{b} B^{*} S$-wave phase shifts with fourchannel coupling for the $I J^{P}=\frac{1}{2} \frac{3}{2}-$ system.

For the hidden-bottom pentaquarks with $I J^{P}=\frac{1}{2} \frac{3}{2}-$, the states $\Sigma_{b} B^{*}$ and $\Sigma_{b}^{*} B$ appear as resonance states in the scattering phase shifts of the $N \Upsilon$ and $\Lambda_{b} B^{*}$ channels. The $\Sigma_{b}^{*} B^{*}$ state appears as a resonance state only in the $\Lambda_{b} B^{*}$ scattering process. All of the phase shifts are shown in Figs. 7 and 8. The masses and decay widths are listed in Table V, from which we can see that the $\Sigma_{b} B^{*}$ state has a mass range of 11 122.2-11 $122.7 \mathrm{MeV}$ and a decay width of $0.4 \mathrm{MeV}$, and the $\Sigma_{b}^{*} B$ state has a mass range of 11 102.4-11 103.6 MeV and a decay width of $1.1 \mathrm{MeV}$. The resonance mass of the $\Sigma_{b}^{*} B^{*}$ state is $11150.0 \mathrm{MeV}$ and its decay width is $1.8 \mathrm{MeV}$.

Similarly, the behavior of the low-energy phase shifts of both $N \eta_{b}$ and $N \Upsilon$ also implies that $N \eta_{b}$ and $N \Upsilon$ with $I J^{P}=\frac{1}{2} \frac{1}{2}^{-}$and $N \Upsilon$ with $I J^{P}=\frac{1}{2} \frac{3}{2}^{-}$can all be bound by channel-coupling calculations.

TABLE V. The masses and decay widths (in $\mathrm{MeV}$ ) of the $I J^{P}=$ $\frac{1}{2} \frac{3}{2}-$ resonance states in the $N \Upsilon$ and $\Lambda_{b} B^{*} S$-wave scattering process.

\begin{tabular}{|c|c|c|c|c|c|c|}
\hline & \multicolumn{6}{|c|}{ Two-channel coupling } \\
\hline & \multicolumn{2}{|c|}{$\Sigma_{b} B^{*}$} & \multicolumn{2}{|c|}{$\Sigma_{b}^{*} B$} & \multicolumn{2}{|c|}{$\Sigma_{b}^{*} B^{*}$} \\
\hline & $M^{\prime}$ & $\Gamma_{i}$ & $M^{\prime}$ & $\overline{\Gamma_{i}}$ & $M^{\prime}$ & $\Gamma_{i}$ \\
\hline$N \Upsilon$ & 11126.3 & 1.7 & 11105.8 & 4.4 & 11155.7 & 3.8 \\
\hline$\Lambda_{b} B^{*}$ & 11125.5 & 0.9 & 11103.5 & 2.6 & 11152.0 & 2.7 \\
\hline \multirow[t]{4}{*}{$\Gamma_{\text {total }}$} & & 2.6 & & 7.0 & & 6.5 \\
\hline & \multicolumn{6}{|c|}{ Four-channel coupling } \\
\hline & \multicolumn{2}{|c|}{$\Sigma_{b} B^{*}$} & \multicolumn{2}{|c|}{$\Sigma_{b}^{*} B$} & \multicolumn{2}{|c|}{$\Sigma_{b}^{*} B^{*}$} \\
\hline & $M^{\prime}$ & $\Gamma_{i}$ & $M^{\prime}$ & $\overline{\Gamma_{i}}$ & $M^{\prime}$ & $\Gamma_{i}$ \\
\hline$N \Upsilon$ & 11122.7 & 0.2 & 11103.6 & 0.8 & $\mathrm{nr}$ & $\ldots$ \\
\hline$\Lambda_{b} B^{*}$ & 11122.2 & 0.2 & 11102.4 & 0.3 & 11150.0 & 1.8 \\
\hline$\Gamma_{\text {total }}$ & & 0.4 & & 1.1 & & 1.8 \\
\hline
\end{tabular}




\section{SUMMARY}

In summary, we investigated the hidden-charm and hidden-bottom pentaquark resonances in the hadronhadron scattering process. For the hidden-charm sector, three resonance states with $I J^{P}=\frac{1}{2} \frac{1}{2}$ and three resonance states with $I J^{P}=\frac{1}{2} \frac{3}{2}-$ were found to be dynamically generated from coupled scattering channels. Because of the hidden $c \bar{c}$ components involved in these states, the masses of these states are all above $4.2 \mathrm{GeV}$ while their widths are only a few MeV. Similar results were obtained for the hidden-bottom system. Both of the resonance states with $I J^{P}=\frac{11}{2} \frac{-}{2}$ and $I J^{P}=\frac{13}{2}{ }^{-}$were found from the corresponding scattering processes. The masses of these states are all above $11 \mathrm{GeV}$ while their widths are only a few $\mathrm{MeV}$. The nature of these states is similar to the corresponding $N_{c \bar{c}}^{*}$ and $N_{b \bar{b}}^{*}$ states predicated in Refs. [4,49], which definitely cannot be accommodated by the conventional $3 q$ quark models, and should form part of the heavy island for the quite stable $N^{*}$ baryons.

In particular, the behavior of the low-energy phase shifts of $N \eta_{c}, N J / \psi, N \eta_{b}$, and $N \Upsilon$ indicates that the states $N \eta_{c}$,
$N J / \psi, N \eta_{b}$, and $N \Upsilon$ with $I J^{P}=\frac{1}{2} \frac{1}{2}$ and $N J / \psi$ and $N \Upsilon$ with $I J^{P}=\frac{1}{2} \frac{3}{2}-$ can all be bound by channel-coupling calculations.

All of these heavy pentaquarks are worth investigating in future experiments. Immediately following the $\mathrm{LHCb}$ Collaboration's claim, the JLab Collaboration proposed searching for hidden-charm pentaquarks by using the photoproduction of $J / \psi$ at threshold in Hall C [50]. Moreover, pentaquarks with charm quarks can also be observed by the PANDA/FAIR experiment [51]. We hope that the proposed Electron-Ion Collider [52] and Jefferson Lab [53] will help to discover these interesting hiddenbottom pentaquarks.

\section{ACKNOWLEDGMENTS}

This work is supported in part by the National Science Foundation of China under Contracts No. 11675080, No. 11775118, and No. 11535005, and the Natural Science Foundation of the Jiangsu Higher Education Institutions of China (Grant No. 16KJB140006).
[1] T. Nakano et al. (LEPS Collaboration), Phys. Rev. Lett. 91, 012002 (2003).

[2] T. Nakano et al. (LEPS Collaboration), Phys. Rev. C 79, 025210 (2009).

[3] M. Naruki (J-PARC E19 Collaboration), Acta Phys. Pol. B 47, 385 (2016).

[4] J. J. Wu, R. Molina, E. Oset, and B. S. Zou, Phys. Rev. Lett. 105, 232001 (2010); Phys. Rev. C 84, 015202 (2011).

[5] Z. C. Yang, Z. F. Sun, J. He, X. Liu, and S. L. Zhu, Chin. Phys. C 36, 6 (2012).

[6] C. W. Xiao, J. Nieves, and E. Oset, Phys. Rev. D 88, 056012 (2013).

[7] T. Uchino, W. H. Liang, and E. Oset, Eur. Phys. J. A 52, 43 (2016).

[8] M. Karliner and J. L. Rosner, Phys. Rev. Lett. 115, 122001 (2015).

[9] E. J. Garzon and J. J. Xie, Phys. Rev. C 92, 035201 (2015).

[10] W. L. Wang, F. Huang, Z. Y. Zhang, and B. S. Zou, Phys. Rev. C 84, 015203 (2011).

[11] S. G. Yuan, K. W. We, J. He, H. S. Xu, and B. S. Zou, Eur. Phys. J. A 48, 61 (2012).

[12] Y. Huang, J. He, H. F. Zhang, and X. R. Chen, J. Phys. G 41, 115004 (2014).

[13] R. Aaij et al. (LHCb Collaboration), Phys. Rev. Lett. 115, 072001 (2015).

[14] R. Chen, X. Liu, X. Q. Li, and S. L. Zhu, Phys. Rev. Lett. 115, 132002 (2015).

[15] H. X. Chen, W. Chen, X. Liu, T. G. Steele, and S. L. Zhu, Phys. Rev. Lett. 115, 172001 (2015).
[16] L. Roca, J. Nieves, and E. Oset, Phys. Rev. D 92, 094003 (2015).

[17] J. He, Phys. Lett. B 753, 547 (2016).

[18] H. X. Huang, C. R. Deng, J. L. Ping, and F. Wang, Eur. Phys. J. C 76, 624 (2016).

[19] G. Yang, J. L. Ping, and F. Wang, Phys. Rev. D 95, 014010 (2017).

[20] U. G. Meissner and J. A. Oller, Phys. Lett. B 751, 59 (2015).

[21] C. W. Xiao and U. G. Meissner, Phys. Rev. D 92, 114002 (2015).

[22] R. Chen, X. Liu, and S. L. Zhu, Nucl. Phys. A954, 406 (2016).

[23] H. X. Chen, E. L. Cui, W. Chen, T. G. Steele, X. Liu, and S. L. Zhu, Eur. Phys. J. C 76, 572 (2016).

[24] K. Azizi, Y. Sarac, and H. Sundu, Phys. Rev. D 95, 094016 (2017).

[25] R. F. Lebed, Phys. Lett. B 749, 454 (2015).

[26] R. Zhu and C. F. Qiao, Phys. Lett. B 756, 259 (2016).

[27] L. Maiani, A. D. Polosa, and V. Riquer, Phys. Lett. B 749, 289 (2015).

[28] V. V. Anisovich, M. A. Matveev, J. Nyiri, A. V. Sarantsev, and A. N. Semenova, arXiv:1507.07652.

[29] R. Ghosh, A. Bhattacharya, and B. Chakrabarti, Phys. Part. Nucl. Lett. 14, 550 (2017).

[30] Z. G. Wang, Eur. Phys. J. C 76, 70 (2016).

[31] A. Mironov and A. Morozov, JETP Lett. 102, 271 (2015).

[32] N. N. Scoccola, D. O. Riska, and M. Rho, Phys. Rev. D 92 , 051501 (2015).

[33] F. K. Guo, U.-G. Meissner, W. Wang, and Z. Yang, Phys. Rev. D 92, 071502(R) (2015). 
[34] X. H. Liu, Q. Wang, and Q. Zhao, Phys. Lett. B 757, 231 (2016).

[35] M. Mikhasenko, arXiv:1507.06552.

[36] U. Skerbis and S. Prelovsek, arXiv:1811.02285.

[37] H. X. Chen, W. Chen, X. Liu, and S. L. Zhu, Phys. Rep. 639, 1 (2016).

[38] K. Azizi, Y. Sarac, and H. Sundu, Phys. Rev. D 96, 094030 (2017).

[39] C. W. Shen, D. Ronchen, U.-G. Meiner, and B. S. Zou, Chin. Phys. C 42, 023106 (2018).

[40] J.-M. Richarda, A. Valcarceb, and J. Vijandec, Phys. Lett. B 774, 710 (2017).

[41] J. L. Ping, H. X. Huang, H. R. Pang, F. Wang, and C. W. Wong, Phys. Rev. C 79, 024001 (2009).

[42] H. Gao, H. Huang, T. Liu, J. Ping, F. Wang, and Z. Zhao, Phys. Rev. C 95, 055202 (2017).

[43] F. Wang, G. H. Wu, L. J. Teng, and T. Goldman, Phys. Rev. Lett. 69, 2901 (1992); G. H. Wu, L. J. Teng, J. L. Ping, F. Wang, and T. Goldman, Phys. Rev. C 53, 1161 (1996).

[44] H. X. Huang, P. Xu, J. L. Ping, and F. Wang, Phys. Rev. C 84, 064001 (2011).
[45] M. Kamimura, Prog. Theor. Phys. Suppl. 62, 236 (1977).

[46] J. L. Ping, F. Wang, and T. Goldman, Nucl. Phys. A657, 95 (1999); G. H. Wu, J. L. Ping, L. J. Teng, F. Wang, and T. Goldman, Nucl. Phys. A673, 279 (2000); H. R. Pang, J. L. Ping, F. Wang, and T. Goldman, Phys. Rev. C 65, 014003 (2001); J. L. Ping, F. Wang, and T. Goldman, Nucl. Phys. A688, 871 (2001); J. L. Ping, H. R. Pang, F. Wang, and T. Goldman, Phys. Rev. C 65, 044003 (2002).

[47] A. Valcarce, H. garcilazo, F. Fernandez, and P. Gonzalez, Rep. Prog. Phys. 68, 965 (2005), and reference therein.

[48] J. Vijande, F. Fernandez, and A. Valcarce, J. Phys. G 31, 481 (2005).

[49] J. J. Wu, L. Zhao, and B. S. Zou, Phys. Lett. B 709, 70 (2012).

[50] Z.-E. Meziani et al. (JLab Collaboration), arXiv:1609 $.00676 \mathrm{v} 2$.

[51] M. F. M. Lutz et al. (PANDA Collaboration), arXiv:0903 .3905.

[52] V. Ptitsyn, AIP Conf. Proc. 735, 1149 (2009).

[53] S. Joosten and Z.-E. Meziani, Proc. Sci. QCDEV2017 (2018) 017. 